\title{
Disrupted Global and Regional Structural Networks and Subnetworks in Children with Localization-Related Epilepsy
}

E. Widjaja, M. Zamyadi, C. Raybaud, O.C. Snead, S.M. Doesburg, and M.L. Smith

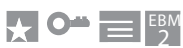

\begin{abstract}
BACKGROUND AND PURPOSE: Structural connectivity has been thought to be a less sensitive measure of network changes relative to functional connectivity in children with localization-related epilepsy. The aims of this study were to investigate the structural networks in children with localization-related epilepsy and to assess the relation among structural connectivity, intelligence quotient, and clinical parameters.
\end{abstract}

MATERIALS AND METHODS: Forty-five children with nonlesional localization-related epilepsy and 28 healthy controls underwent DTI. Global network (network strength, clustering coefficient, characteristic path length, global efficiency, and small-world parameters), regional network (nodal efficiency), and the network-based statistic were compared between patients and controls and correlated with intelligence quotient and clinical parameters.

RESULTS: Patients showed disrupted global network connectivity relative to controls, including reduced network strength, increased characteristic path length and reduced global efficiency, and reduced nodal efficiency in the frontal, temporal, and occipital lobes. Connectivity in multiple subnetworks was reduced in patients, including the frontal-temporal, insula-temporal, temporal-temporal, frontal-occipital, and temporal-occipital lobes. The frontal lobe epilepsy subgroup demonstrated more areas with reduced nodal efficiency and more impaired subnetworks than the temporal lobe epilepsy subgroup. Network parameters were not significantly associated with intelligence quotient, age at seizure onset, or duration of epilepsy.

CONCLUSIONS: We found disruption in global and regional networks and subnetworks in children with localization-related epilepsy. Regional efficiency and subnetworks were more impaired in frontal lobe epilepsy than in temporal lobe epilepsy. Future studies are needed to evaluate the implications of disrupted networks for surgical resection and outcomes for specific epileptogenic zones and the relation of disrupted networks to more complex cognitive function.

ABBREVIATIONS: $\mathrm{AAL}=$ automated anatomic labeling; FLE = frontal lobe epilepsy; IQ = intelligence quotient; NBS = network-based statistic; TLE = temporal lobe epilepsy

E pilepsy has been considered a disorder of neural networks, in which activity in one part of the network is influenced by activity elsewhere in the network. ${ }^{1}$ The epileptogenic network could

Received September 21, 2014; accepted after revision January 2, 2015.

From the Department of Diagnostic Imaging (E.W., M.Z., C.R., S.M.D.), Division of Neurology (E.W., O.C.S.), and Neuroscience and Mental Health at Research Institute (S.M.D.), Hospital for Sick Children, Toronto, Ontario, Canada; and Department of Psychology (M.L.S.), University of Toronto, Toronto, Ontario, Canada.

This work was supported by SickKids Foundation/Canadian Institutes of Health Research, Institute of Human Development, Child and Youth Health, and the Derek Harwood Nash Chair fund.

Please address correspondence to E. Widjaja, MD, 555 University Ave, Toronto, ON M5G 1X8, Canada; e-mail: Elysa.Widjaja@sickkids.ca

-- Indicates open access to non-subscribers at www.ajnr.org

Indicates article with supplemental on-line appendix and tables.

Evidence-Based Medicine Level 2.

http://dx.doi.org/10.3174/ajnr.A4265 be responsible for the development and spread of seizures or could develop secondary to reorganization of the normal neural networks from recurrent seizures. Children with localization-related epilepsy, including those with frontal lobe epilepsy (FLE), have demonstrated abnormal resting-state functional connectivity in the default mode network and in other resting-state networks, such as attention, frontal, visual, auditory, and somatosensory networks. ${ }^{2,3}$ Graph theoretical measures of functional connectivity have also been shown to be abnormal in children with FLE, particularly in those who were cognitively impaired, with increased path length, an increased weighted cluster coefficient, and higher modularity, implying that the networks are less integrated and more segregated. ${ }^{4}$

Less is known about structural connectivity in children with localization-related epilepsy other than FLE. DTI measures of WM microstructural organization such as fractional anisotropy 
and mean diffusivity have been shown to be impaired in children with localization-related epilepsy, also known as partial or focal epilepsy, including FLE and temporal lobe epilepsy (TLE). ${ }^{5-9}$ These changes were identified not only in the WM ipsilateral to seizure focus but also contralateral to the seizure focus. Given that there was bilateral structural abnormality in children with localization-related epilepsy, we postulated that there would be extensive impairment in structural connectivity despite the apparent focality of the seizure disorders. A recent study in children with FLE $^{10}$ found that structural path length and clustering coefficient assessed by using DTI were normal but the functional connectivity measures assessed by using resting-state fMRI were abnormal; these findings suggest that structural connectivity may be less sensitive than functional connectivity assessment of the networks. It is not known whether the previously reported lack of changes in structural connectivity is specific to FLE and whether there are differences in structural connectivity across different types of localization-related epilepsy, because FLE involves different epileptogenic networks compared with TLE.

The aims of this study were to investigate the structural wholebrain networks by using graph theoretical measures in children with localization-related epilepsy relative to healthy controls and to assess whether there was a relation between graph measures of structural connectivity, intelligence quotient (IQ), and clinical parameters. The secondary aims of this study were to determine whether there were structural network differences between subgroups with FLE and TLE and the relations of graph measures of structural connectivity with IQ and clinical parameters.

\section{MATERIALS AND METHODS}

\section{Subjects}

This study had the approval of the research ethics board of the Hospital for Sick Children. Informed consent was obtained from parents, and assent, from children for both patients and healthy controls. Children with localization-related epilepsy who were worked up for epilepsy surgery were recruited into the study. All patients had normal 3T MR imaging findings. Location of the epileptogenic zone was determined by ictal and interictal video electroencephalography, magnetoencephalography, and FDGPET scans. The control group consisted of healthy children with no neurologic or psychiatric disorders and normal MR imaging findings. Both patients and controls underwent the Wechsler Abbreviated Scale of Intelligence to assess IQ.

\section{MR Imaging and DTI}

MR imaging was performed on a 3T scanner (Achieva; Philips Healthcare, Best, the Netherlands) by using an 8-channel phased array head coil in all subjects. The imaging in patients consisted of axial and coronal FLAIR, proton-attenuation and T2, and volumetric $\mathrm{T} 1(\mathrm{TR} / \mathrm{TE}=4.9 / 2.3 \mathrm{~ms}$, section thickness $=1 \mathrm{~mm}$, FOV $=22 \mathrm{~cm}$, matrix $=220 \times 220$, scan time $=6$ minutes 13 seconds), and DTI (TR/TE $=10,000 / 60 \mathrm{~ms}$, section thickness $=2$ $\mathrm{mm}, \mathrm{FOV}=22 \mathrm{~cm}$, matrix $=112 \times 112, \mathrm{NEX}=1$, sensitivity encoding $=2, b=1000 \mathrm{~s} / \mathrm{mm}^{2}, 31$ noncollinear directions, number of sections $=70$, isotropic voxel $=2 \mathrm{~mm}$, scan time $=6$ minutes 41 seconds). The imaging in controls included volumetric $\mathrm{T} 1$ and DTI, by using the same parameters as those for patients.
There were no significant differences in relative displacement between consecutive gradient images $(P=.080)$ and translational $(P=.0704)$ and rotational $(P=.2339)$ motion between patients and controls.

\section{Data Preprocessing}

The DTI data were preprocessed by using fMRI of the Brain Software Library tools (Version 5.0, http://www.fmrib.ox.ac.uk/fsl). The raw diffusion tensor images were corrected for head motion and eddy current distortion. ${ }^{11}$ To achieve this, we aligned all 31 diffusion-weighted images to the first B0 scan by using affine registration. The set of gradient vectors of each subject was then adjusted according to the affine transformation of the individual images.

\section{Tractography}

Tractography was initiated from seeds on the boundary of the gray-white matter interface. ${ }^{4,12}$ The FMRIB Automated Segmentation Tool (http://fsl.fmrib.ox.ac.uk/fsl/fslwiki/fast) ${ }^{13}$ was used to segment the individual skull-stripped structural T1 images into gray and white matter. The deep gray matter was manually removed from the whole-brain boundary mask. The seed voxels in the gray-white matter boundary were then transformed into each subject's native DTI space by using nonlinear coregistration.

The Camino toolbox ${ }^{14}$ (www.camino.org.uk) was then used to perform probabilistic tractography in the native DTI space. A Bayesian approach ${ }^{15}$ was used to initiate the fiber tracking from the gray-white matter boundary of the whole brain. This approach models the uncertainty in WM tractography by computing the likelihood of the fiber orientation by drawing a principal direction from a posterior distribution once per iteration for each seed voxel. The number of iterations at each seed voxel was set to 1000 .

\section{Network Construction}

The automated anatomic labeling (AAL) template was used to define 82 cortical regions ${ }^{16}$ in each subject. ${ }^{17}$ The AAL ROIs were transformed to the native DTI space of each subject by using the following steps: First, both unweighted B0 and structural T1 images were skull-stripped, and nonlinear co-registration was preformed between the T1 and B0 image by using the 3DSlicer (http://www.slicer.org/). ${ }^{18}$ Next, the Montreal Neurological Institute 152 brain template was nonlinearly registered to each individual's structural T1 image by using the Advanced Normalization Tools software package (http://stnava.github.io/ANTs/). The transformations from these 2 steps were then concatenated and used to resample the AAL atlas in the Montreal Neurological Institute space into each subject's native DTI space. Subsequently, the DTI of each subject was parcellated into 82 cortical ROIs. Each AAL ROI represented a node in the graph representation of the structural network. To construct the structural network, we extracted fiber bundles connecting each pair of AAL ROIs from the entire set of whole-brain tracts. A connectivity probability $P_{\mathrm{ij}}$ from a seed region $i$ with $n$ number of voxels to another region $j$ was calculated by dividing the number of fibers passing through region $j$ by $1000 \times n \cdot{ }^{17,19}$ Next, a weighted undirected $82 \times 82$ connectivity matrix was constructed for each subject. 
Table 1: Characteristics of patients with epilepsy, including those with frontal lobe epilepsy and temporal lobe epilepsy and controls

\begin{tabular}{lcccc}
\hline & $\begin{array}{c}\text { Controls } \\
(\boldsymbol{n}=\mathbf{2 8})\end{array}$ & $\begin{array}{c}\text { All Epilepsy } \\
(\boldsymbol{n}=\mathbf{4 5})\end{array}$ & $\begin{array}{c}\text { FLE } \\
(\boldsymbol{n}=\mathbf{2 5})\end{array}$ & $\begin{array}{c}\text { TLE } \\
(\boldsymbol{n}=16)\end{array}$ \\
\hline Age (yr) & $13.8(3.1)$ & $13.6(3.0)$ & $13.1(3.3)$ & $14.4(2.7)$ \\
Age at seizure onset $(\mathrm{yr})$ & $\mathrm{NA}$ & $8.4(3.9)$ & $8.0(4.0)$ & $9.2(3.5)$ \\
Duration of epilepsy $(\mathrm{yr})$ & $\mathrm{NA}$ & $5.1(3.2)$ & $5.1(2.9)$ & $4.9(3.7)$ \\
No. of antiepileptic medications & NA & $2.0(0.7)$ & $2.0(0.7)$ & $2.0(0.7)$ \\
IQ & $112.8(10.8)$ & $97.0(11.9)$ & $97.6(10.9)$ & $97.6(11.9)$ \\
\hline
\end{tabular}

Note:-NA indicates not applicable.

\section{Graph Theoretical Analysis}

The Matlab-based Brain Connectivity Toolbox (http://www. brain-connectivity-toolbox.net/ ${ }^{20}$ was used to perform graph theoretical network analysis to investigate potential differences in brain structural network parameters between patients with epilepsy and healthy controls. The metrics assessed included network strength, clustering coefficient, characteristic path length, global efficiency, small-world parameter, and nodal efficiency (On-line Appendix). The network strength is defined as the average of the strengths over all nodes in the graph. The clustering coefficient reflects functional segregation of brain networks, which is a measure of the propensity of the brain to execute specialized processes within interconnected groups of brain regions or clusters. ${ }^{20} \mathrm{~A}$ high clustering coefficient indicates that the neighbors of a node are directly connected to each other. ${ }^{21}$ The characteristic path length is the most commonly used measure of functional integration and quantifies the ability of the network to propagate information in parallel. ${ }^{20}$ Global efficiency reflects how efficiently information can be exchanged over the whole network, with larger global efficiency indicating a more efficient network. Small-world networks are defined as networks that have a characteristic path length similar to that of random networks but are significantly more clustered than random networks. ${ }^{21}$ Scalar measure of small-world property $(\sigma)>1$ indicates that the network has a small-world property. The nodal efficiency quantifies the importance of the nodes for communication within the network. The nodes with high nodal efficiencies can be categorized as hubs in a network. ${ }^{22}$

\section{Network-Based Statistic}

A network-based statistic (NBS) ${ }^{23}$ was used to identify clusters of nodes and edges (subnetworks) in a connectivity matrix, which were differentially connected between patients and controls. First, significant nonzero connections within each group that were common across all subjects were detected, and then NBS (http:// www.nitrc.org/projects/nbs/) was applied to those connections. A set of superthreshold links (ie, edges) was defined by applying a primary threshold ( $P=.01)$ to the test statistics (2-sample, 1-tailed $t$ test) computed for each link. Any connected components and their number of links (ie, component size) were then selected among the superthreshold links. Finally, a nonparametric permutation test (10,000 permutations) and a secondary threshold of $P=.05$ were used to choose the significant components. ${ }^{24-26}$ This method effectively controls for false-positives due to multiple comparisons.

\section{Statistical Analysis}

Comparison of Network Characteristics. Differences in network characteristics (network strength, clustering coefficient, characteristic path length, global efficiency, small-world parameters, and nodal efficiency) between patients with epilepsy and controls were assessed by using 2-sample $t$ tests. Subsequently, subgroup analyses comparing network characteristics of FLE, TLE, and controls were performed by using 2-sample $t$ tests.

Correlation of Clinical Parameters with Network Characteristics. Partial correlation analyses were performed to assess the relation among network characteristics, IQ, and clinical parameters (age at seizure onset and duration of epilepsy), controlling for age and sex, in all patients with epilepsy and the subgroups with FLE and TLE.

\section{RESULTS}

\section{Subjects}

Forty-five children with nonlesional localization-related epilepsy and 28 healthy controls were recruited. The mean age of patients (13.6 \pm 3.0 years; range, $7.0-19.1$ ) was not significantly different from the mean age of controls ( $13.8 \pm 3.1$ years; range, $7.0-18.8$ years) $(P=.856)$ (Table 1$)$. The mean age of seizure onset was $8.4 \pm 3.9$ years, the mean duration of epilepsy was $5.1 \pm 3.2$ years, and the mean number of antiepileptic medications was $2.0 \pm 0.7$. The mean IQ of patients $(97.0 \pm 11.9)$ was significantly lower than that of controls $(112.8 \pm 10.8)(P<.001)$. Twenty-five patients had FLE, 17 with left FLE and 8 with right FLE. Sixteen patients had TLE, 12 with left TLE and 4 with right TLE. Two patients had right hemisphere, 1 had right parietal, and another had right parieto-occipital seizure onset. There were no significant differences in age, age at seizure onset, duration of epilepsy, number of antiepileptic medications, and IQ of patients with FLE versus TLE (all, $P>.05$ ).

\section{Alterations in Global Properties of Structural Networks}

All Epilepsy versus Controls. The network parameters are shown in Table 2. The network strength was significantly decreased in patients $(P=.0002)$, but there was no significant change in the clustering coefficient $(P=.8034)$ of patients relative to controls. The characteristic path length was significantly increased $(P<$ $.0001)$, and the global efficiency was significantly decreased $(P=$ $.0003)$ in patients relative to controls. Patients and controls demonstrated small-world organization of the WM networks $(\sigma>1)$. The small-world property $(\sigma)$ was greater $(P=.0353)$ in patients than in controls.

FLE versus Controls. The network strength was significantly decreased in patients with $\operatorname{FLE}(P=.0001)$, but there was no significant difference in the clustering coefficient $(P=.8034)$ of patients with FLE relative to controls. The characteristic path length was significantly increased $(P<.0001)$, and the global efficiency was significantly decreased $(P<.0001)$ in patients with FLE. Pa- 
Table 2: Global properties of structural networks of controls and all patients with epilepsy, frontal lobe epilepsy, and temporal lobe epilepsy relative to controls

\begin{tabular}{|c|c|c|c|c|}
\hline & Controls & All Epilepsy & FLE & TLE \\
\hline Network strength \pm SD & $0.7183 \pm 0.0249$ & $0.6960 \pm 0.0226(P=.0002)^{a}$ & $0.6900 \pm 0.0244(P=.00012)^{a}$ & $0.7049 \pm 0.0189(P=.0641)$ \\
\hline Clustering coefficient \pm SD & $0.0041 \pm 0.0004$ & $0.0041 \pm 0.0005(P=.8034)$ & $0.0040 \pm 0.0005(P=.3091)$ & $0.0041 \pm 0.0004(P=.6662)$ \\
\hline Characteristic path length $\pm S D$ & $32.0769 \pm 1.1222$ & $33.3724 \pm 1.3593(P<.0001)^{\mathrm{a}}$ & $33.6940 \pm 1.4205(P<.0001)^{\mathrm{a}}$ & $33.0110 \pm 1.2915(P=.0142)^{\mathrm{a}}$ \\
\hline Global efficiency \pm SD & $0.0419 \pm 0.0013$ & $0.0407 \pm 0.0014(P=.0003)^{a}$ & $0.0403 \pm 0.0015(P<.0001)^{\mathrm{a}}$ & $0.0412 \pm 0.0013(P=.0613)$ \\
\hline Small-world property \pm SD & $2.0018 \pm 0.1435$ & $2.0772 \pm 0.1483(P=.0353)^{\mathrm{a}}$ & $2.0646 \pm 0.1562(P=.1335)$ & $2.0750 \pm 0.1374(P=.0992)$ \\
\hline
\end{tabular}

tients with FLE demonstrated small-world organization of the WM networks $(\sigma>1)$. There was no significant difference in small-world property $(P=.1335)$ in patients with FLE relative to controls.

TLE versus Controls. There was a trend toward reduced network strength $(P=.0641)$ and no significant difference in clustering coefficient $(P=.6662)$ of patients with TLE relative to controls. The characteristic path length was significantly increased $(P=$ .0142 ), and there was a trend toward reduced global efficiency $(P=.0613)$ in patients with TLE relative to controls. Patients with TLE demonstrated small-world organization of the WM networks $(\sigma>1)$. There was no significant difference in small-world property $(P=.0992)$ in patients with TLE relative to controls.

FLE versus TLE. The network strength was significantly decreased $(P=.0399)$, and there was a trend toward reduced global efficiency $(P=.0596)$ in patients with FLE relative to those with TLE. There was no significant difference in clustering coefficient $(P=$ .2216), characteristic path length $(P=.1208)$, and small-world property $(P=.8250)$ of patients with FLE relative to those with TLE.

\section{Alterations in Regional Properties of Structural Networks}

All Epilepsy versus Controls. Thirty-three regions showed reduced nodal efficiency $(P<.05)$ in patients with epilepsy compared with controls, in the frontal, temporal, and occipital lobes (Fig 1 and On-line Table 1). The right anterior cingulate, right and left amygdalae, hippocampi, parahippocampal gyri, and superior temporal gyri showed a more statistically reliable reduction $(P<$ .001 ) in nodal efficiency.

FLE versus Controls. Fifty-one regions showed reduced nodal efficiency $(P<.05)$ in patients with FLE compared with controls, in the frontal, temporal, and occipital lobes. The right and left parahippocampal gyri, amygdalae, fusiform and superior temporal gyri, and right middle temporal gyrus showed a more statistically reliable reduction $(P<.001)$ in nodal efficiency.

TLE versus Controls. Fourteen regions showed reduced nodal efficiency $(P<.05)$ in patients with TLE compared with controls, in the frontal, temporal, and occipital lobes. The right and left amygdalae and right superior temporal gyrus showed a more statistically reliable reduction $(P<.001)$ in nodal efficiency.

FLE versus TLE. Sixteen regions showed reduced nodal efficiency $(P<.05)$ in patients with FLE relative to those with TLE, in the frontal, temporal, and occipital lobes. None of these regions showed a more statistically reliable reduction $(P<.001)$ in nodal efficiency.
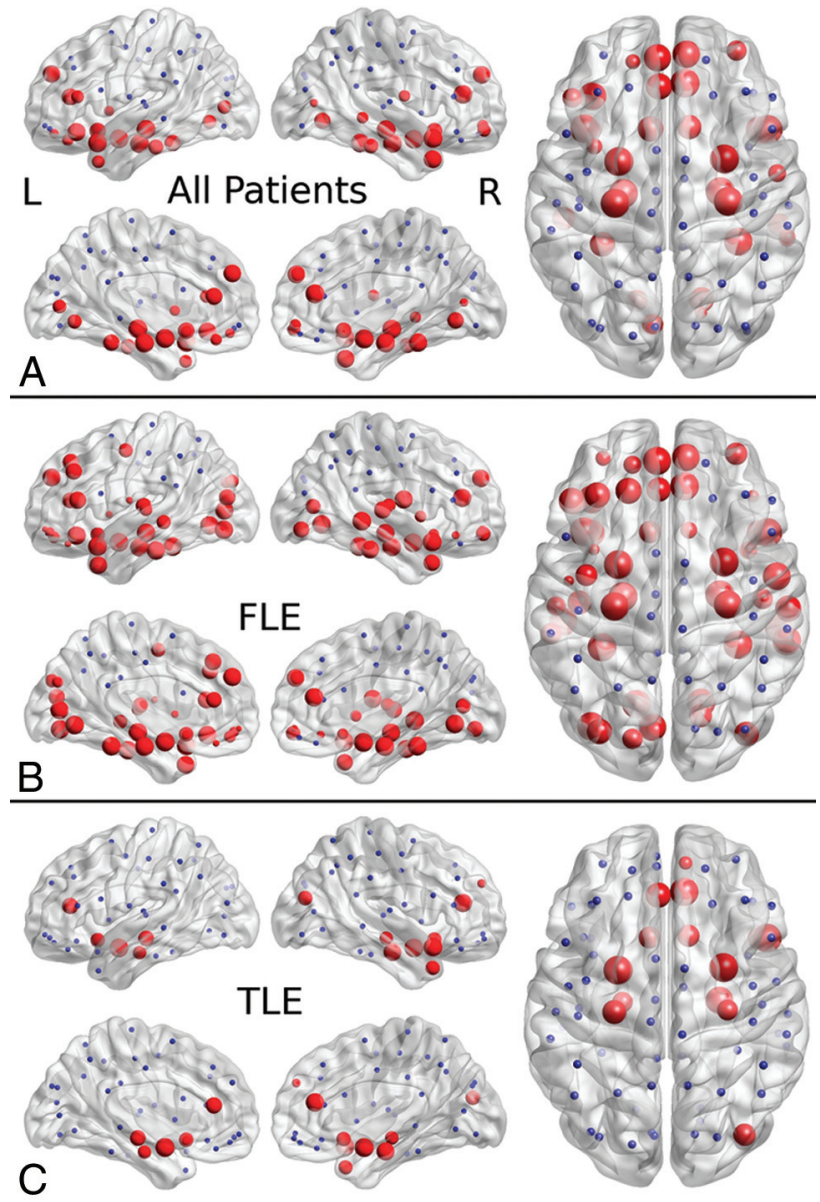

FIG 1. Reduced nodal efficiency in localization-related epilepsy $(A)$, frontal lobe epilepsy $(B)$, and temporal lobe epilepsy $(C)$ in patients relative to controls. The disrupted nodes with reduced nodal efficiency are shown in red, and the unaffected nodes are shown in blue. The size of the nodes is related to the significance of between-group differences in nodal efficiency, with larger nodes representing more significant (lower $P$ value) reduced nodal efficiency in patients relative to controls.

\section{Network-Based Statistic}

All Epilepsy versus Controls. Sixty-one connections demonstrated reduced connectivity $(P<.05$, corrected) in patients relative to controls, involving the frontal-temporal, insula-temporal, temporal-temporal, frontal-occipital, and temporal-occipital lobes (Fig 2 and On-line Table 2). None of the connections showed increased connectivity in patients relative to controls.

FLE versus Controls. Twenty connections demonstrated reduced connectivity $(P<.05$, corrected $)$ in patients with FLE relative to controls, involving the frontal-temporal, insula-temporal, temporal-temporal, temporal-occipital, and occipital-occipital lobes.

AJNR Am J Neuroradiol 36:1362-68 Jul 2015 www.ajnr.org

1365 



TLE
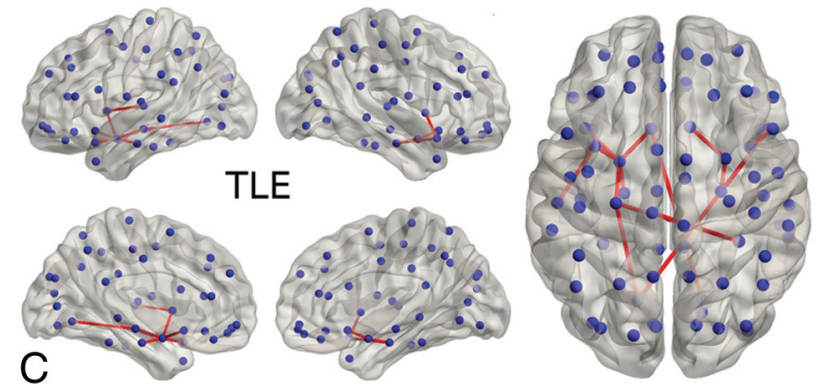

FIG 2. Results of the network-based statistic analysis of the following: $A$, Patients with localization-related epilepsy relative to controls, showing reduced connectivity involving the frontal-temporal, insulatemporal, temporal-temporal, frontal-occipital, and temporal-occipital lobes. B, Patients with frontal lobe epilepsy relative to controls, showing reduced connectivity involving the frontal-temporal, insulatemporal, temporal-temporal, temporal-occipital, and occipital-occipital lobes. C, Patients with temporal lobe epilepsy relative to controls, showing reduced connectivity involving the frontal-temporal, insula-temporal, temporal-temporal, temporal-occipital, and frontaloccipital lobes.

None of the connections showed increased connectivity in patients relative to controls.

TLE versus Controls. Fifteen connections demonstrated reduced connectivity $(P<.05$, corrected $)$ in patients with TLE relative to controls, involving the frontal-temporal, insula-temporal, temporal-temporal, temporal-occipital, and frontal-occipital lobes. None of the connections showed increased connectivity in patients relative to controls.

FLE versus TLE. One hundred forty-two connections demonstrated reduced connectivity, and 26 connections showed increased connectivity $(P<.05$, corrected) in patients with FLE relative to those with TLE.

\section{Correlation with IQ and Clinical Parameters}

All Epilepsy. There was no significant correlation between global network properties and IQ, age at seizure onset, or duration of epilepsy (all, $P>.05$ ) in patients.
FLE. There was no significant correlation between global network properties and IQ, age at seizure onset, or duration of epilepsy (all, $P>.05)$ in patients with FLE.

TLE. There was no significant correlation between global network properties and IQ, age at seizure onset, or duration of epilepsy (all, $P>.05)$ in patients with TLE.

\section{DISCUSSION}

The findings of this study add to the literature by demonstrating impaired structural connectivity affecting global and regional networks and subnetworks in children with localization-related epilepsy, and the impairment in structural connectivity was extensive despite the apparent focality of the seizure disorders. To our knowledge, this is the first study that has compared the structural connectivity in FLE and TLE subgroups, and we found differences between the 2 subgroups, with more regions of reduced nodal efficiency and reduced subnetworks in FLE than in TLE. There was no significant correlation between network characteristics with IQ, age at seizure onset, or duration of epilepsy in patients.

Impairment in global functional integration, as indicated by elevated characteristic path length and decreased global efficiency, suggests that altered architecture of structural brain networks may negatively impact the ability of the brain to rapidly combine specialized information from distributed brain regions coupled with reduced efficiency of the WM networks in children with localization-related epilepsy. This reduction in integration was identified in both FLE and TLE subgroups, suggesting that impaired global functional integration is not specific to subtypes of epilepsy. There was no significant difference in the clustering coefficient between patients with epilepsy and controls, indicating that the extent of local interconnectivity in the network was preserved in the former group. ${ }^{21}$ Vaessen et $\mathrm{al}^{27}$ also found increased path length and decreased clustering coefficient in adult patients with epilepsy with cognitive impairment relative to controls but not in those without cognitive impairment. In a subsequent study, the same authors ${ }^{10}$ showed that there were no significant differences in clustering coefficients, path length, and modularity of the WM connectivity in children with FLE relative to controls. Differences in findings could be related to different populations of FLE, with our patient population having a wider age range and possibly more severe epilepsy relative to the patients in the study by Vaessen et al. ${ }^{10}$ Disruption in the global structural network has also been identified in childhood absence epilepsy ${ }^{28}$ and adults with TLE. ${ }^{29,30}$

Patients with localization-related epilepsy showed reduced regional efficiency affecting multiple regions in the frontal, temporal, and occipital lobes. Liu et $\mathrm{al}^{30}$ also found that adult patients with left mesial TLE have significantly reduced regional efficiency in multiple regions in the temporal, frontal, and parietal lobes. We have found more regions of reduced nodal efficiency in the FLE than in the TLE subgroup, which may be due to the rapid spread of seizure activity as a result of the extensive network of connections of the frontal lobe and more frequent seizures in $\mathrm{FLE}^{31-33}$ or, alternatively, an inherently more extensive abnormal regional efficiency from an underlying etiology that predispose to FLE. The more extensive impaired regional networks may be associated 
with poorer seizure-free surgical outcome in patients with FLE relative to those with TLE. ${ }^{33}$

In this study, we found that children with localization-related epilepsy have extensive reduction in connectivity affecting the frontal-temporal, insula-temporal, temporal-temporal, frontaloccipital, and temporal-occipital lobes, indicating that multiple subnetworks are perturbed, despite the apparent focality of the seizure disorders. The WM tracts affected would include association fibers such as the cingulum, uncinate fasciculus, superior and inferior longitudinal fasciculus, and arcuate fasciculus, as well as the commissural fibers such as the corpus callosum, anterior commissure, and hippocampal commissure. There were more subnetworks expressing reduced structural connectivity in FLE than in TLE, probably related to the rapid spread of seizure activity and more frequent seizures in FLE. ${ }^{31-33}$ In both subgroups, the reduced structural connectivity predominantly involved the mesial temporal and frontal basal structures compared with controls. The spread of seizures from the frontal to temporal lobes in FLE and from the temporal to frontal lobes in TLE may have contributed to reduce structural connectivity between the frontal and temporal lobes. There were more abnormal subnetworks involving the temporal than frontal lobes in those with FLE and also more abnormal subnetworks in the temporal lobes in those with FLE than in those with TLE. Although the reason for this finding is not entirely clear, reorganization of the subnetworks, due to either the spread of seizures or pre-existing abnormal subnetworks that facilitate the spread of seizures to the temporal lobes, compounded by more frequent seizures in FLE, may have contributed to the observation. Adult patients with TLE have also shown reduced structural connectivity between the temporal and frontal cortices, including the orbitofrontal cortex. ${ }^{29,30}$ The WM tracts connecting the frontal lobe to the temporal and occipital lobes have demonstrated smaller volumes in children with FLE compared with controls, ${ }^{34}$ indicating impaired structural connections between the frontal and temporal and occipital lobes.

Vaessen et $\mathrm{al}^{4}$ found that the clustering coefficient was significantly positively correlated with IQ, and path length was negatively correlated with IQ in adults with cryptogenic localizationrelated epilepsy. In that study, ${ }^{4}$ the patients had a broader range of IQ scores including those who are nonimpaired (mean IQ of 97) and those who are impaired (mean IQ of 85). In a subsequent study, ${ }^{10}$ the same authors did not find an association between the clustering coefficient and path length measures of structural or functional connectivity with a computerized visual searching task in children with FLE. Liu et $\mathrm{al}^{30}$ did not find any association among characteristic path length, global efficiency, and local efficiency with neurocognitive function including IQ, auditory verbal learning, and visual memory, as well as with age at seizure onset and duration of epilepsy in adults with left mesial TLE. We did not find any associations between graph network measures and IQ scores. It is possible that with the narrower range of IQs in our study population, we were not able to identify a correlation between graph measures and IQ. Alternatively, the disrupted global structural network may be associated with more complex cognitive processing beyond IQ or a computerized visual searching task.

There are several limitations in this study. We have used an
AAL template to define the ROIs, which was developed from a single adult brain. Therefore, it is possible that the ROI positioning may be less precise in children due to differences in head size. The AAL template has been used in children with absence epilepsy, ${ }^{28}$ and similar finding of a disrupted global structural network was found. We have categorized the patients to the 2 main subgroups of FLE or TLE. Some heterogeneity in the epileptogenic zone was present within each subgroup, with the TLE subgroup comprising mesial and lateral TLE and the FLE subgroup comprising the epileptogenic zone in the motor or supplementary motor cortex, focal within a specific gyrus or more diffuse involvement within the frontal lobe. Due to the small sample size within each specific epileptogenic zone, we have opted to combine the patients into 2 main subgroups. Despite the heterogeneity in the subgroups, we have found differences in regional efficiency and subnetworks between FLE and TLE. Future study evaluating networks of individual patients may help clarify the differences between epileptogenic zones.

\section{CONCLUSIONS}

We found disruption in global structural networks, reduced regional efficiency in multiple lobes, and extensive reduction in subnetwork connectivity in children with localization-related epilepsy, indicating widespread disruption in structural connectivity despite the apparent focality of the seizure disorders. This disruption in structural connectivity may have been responsible for the development and propagation of seizures or may be a secondary phenomenon due to recurrent seizures reorganizing the networks or alternatively due to an underlying etiology that perturbs the neural networks and also causes seizures. Those with FLE demonstrated more areas with reduced regional efficiency and more subnetworks expressing reduced structural connectivity than did those with TLE. Future study evaluating networks of individual patients may help clarify the nuances of regional networks and subnetworks for specific epileptogenic zones and the implications of these networks on surgical resection and outcomes. We did not find any associations between global network properties and IQ. Further studies of patients with a wider range of IQs and assessing more complex cognitive function are needed to evaluate the relation between measures of global network properties and cognitive function.

Disclosures: Elysa Widjaja—RELATED: Grant: SickKids Foundation/Canadian Institutes of Health Research, Institute of Human Development, Child and Youth Health*; Derek Harwood Nash Chair fund*; UNRELATED: Grants/Grants Pending: Canadian Institute of Health Research, ${ }^{*}$ Ontario Brain Institute. * * Money paid to the institution.

\section{REFERENCES}

1. Spencer SS. Neural networks in human epilepsy: evidence of and implications for treatment. Epilepsia 2002;43:219-27

2. Widjaja E, Zamyadi M, Raybaud C, et al. Impaired default mode network on resting-state fMRI in children with medically refractory epilepsy. AJNR Am J Neuroradiol 2013;34:552-57

3. Widjaja E, Zamyadi M, Raybaud C, et al. Abnormal functional network connectivity among resting-state networks in children with frontal lobe epilepsy. AJNR Am J Neuroradiol 2013;34:2386-92

4. Vaessen MJ, Jansen JF, Vlooswijk MC, et al. White matter network abnormalities are associated with cognitive decline in chronic epilepsy. Cereb Cortex 2012;22:2139-47

AJNR Am J Neuroradiol 36:1362-68 Jul 2015 www.ajnr.org 
5. Widjaja E, Skocic J, Go C, et al. Abnormal white matter correlates with neuropsychological impairment in children with localizationrelated epilepsy. Epilepsia 2013;54:1065-73

6. Widjaja E, Kis A, Go C, et al. Bilateral white matter abnormality in children with frontal lobe epilepsy. Epilepsy Res 2014;108:289-94

7. Nilsson D, Go C, Rutka JT, et al. Bilateral diffusion tensor abnormalities of temporal lobe and cingulate gyrus white matter in children with temporal lobe epilepsy. Epilepsy Res 2008;81:128-35

8. Kim H, Harrison A, Kankirawatana P, et al. Major white matter fiber changes in medically intractable neocortical epilepsy in children: a diffusion tensor imaging study. Epilepsy Res 2013;103:211-20

9. Holt RL, Provenzale JM, Veerapandiyan A, et al. Structural connectivity of the frontal lobe in children with drug-resistant partial epilepsy. Epilepsy Behav 2011;21:65-70

10. Vaessen MJ, Jansen JF, Braakman HM, et al. Functional and structural network impairment in childhood frontal lobe epilepsy. PLoS One 2014;9:e90068

11. Jenkinson M, Smith S. A global optimisation method for robust affine registration of brain images. Med Image Anal 2001;5:143-56

12. Vaessen MJ, Hofman PA, Tijssen HN, et al. The effect and reproducibility of different clinical DTI gradient sets on small world brain connectivity measures. Neuroimage 2010;51:1106-16

13. Zhang Y, Brady M, Smith S. Segmentation of brain MR images through a hidden Markov random field model and the expectationmaximization algorithm. IEEE Trans Med Imaging 2001;20:45-57

14. Cook PA, Bai Y, Nedjati-Gilani S, et al. Camino: open-source diffusion-MRI reconstruction and processing. In: Proceedings of the 14th Scientific Meeting of the International Society for Magnetic Resonance in Medicine, Seattle, Washington. May 6-12, 2006:2759

15. Friman O, Farneback G, Westin CF. A Bayesian approach for stochastic white matter tractography. IEEE Trans Med Imaging 2006;25:965-78

16. Tzourio-Mazoyer N, Landeau B, Papathanassiou D, et al. Automated anatomical labeling of activations in SPM using a macroscopic anatomical parcellation of the MNI MRI single-subject brain. Neuroimage 2002;15:273-89

17. Gong G, Rosa-Neto P, Carbonell F, et al. Age- and gender-related differences in the cortical anatomical network. J Neurosci 2009; 29:15684-93

18. Fedorov A, Beichel R, Kalpathy-Cramer J, et al. 3D Slicer as an image computing platform for the Quantitative Imaging Network. Magn Reson Imaging 2012;30:1323-41

19. Cao Q, Shu N, An L, et al. Probabilistic diffusion tractography and graph theory analysis reveal abnormal white matter structural connectivity networks in drug-naive boys with attention deficit/hyperactivity disorder. J Neurosci 2013;33:10676-87

20. Rubinov M, Sporns O. Complex network measures of brain connectivity: uses and interpretations. Neuroimage 2010;52:1059-69

21. Watts DJ, Strogatz SH. Collective dynamics of 'small-world' networks. Nature 1998;393:440-42

22. Achard S, Bullmore E. Efficiency and cost of economical brain functional networks. PLoS Comput Biol 2007;3:e17

23. Zalesky A, Fornito A, Bullmore ET. Network-based statistic: identifying differences in brain networks. Neuroimage 2010;53:1197-207

24. Zhang J, Wang J, Wu Q, et al. Disrupted brain connectivity networks in drug-naive, first-episode major depressive disorder. Biol Psychiatry 2011;70:334-42

25. Bai F, Shu N, Yuan Y, et al. Topologically convergent and divergent structural connectivity patterns between patients with remitted geriatric depression and amnestic mild cognitive impairment. J Neurosci 2012;32:4307-18

26. Zalesky A, Fornito A, Seal ML, et al. Disrupted axonal fiber connectivity in schizophrenia. Biol Psychiatry 2011;69:80-89

27. Vaessen MJ, Braakman HM, Heerink JS, et al. Abnormal modular organization of functional networks in cognitively impaired children with frontal lobe epilepsy. Cereb Cortex 2013;23: 1997-2006

28. Xue K, Luo C, Zhang D, et al. Diffusion tensor tractography reveals disrupted structural connectivity in childhood absence epilepsy. Epilepsy Res 2014;108:125-38

29. DeSalvo MN, Douw L, Tanaka N, et al. Altered structural connectome in temporal lobe epilepsy. Radiology 2014;270:842-48

30. Liu M, Chen Z, Beaulieu C, et al. Disrupted anatomic white matter network in left mesial temporal lobe epilepsy. Epilepsia 2014; 55:674-82

31. Talairach J, Tournoux P, Musolino A, et al. Stereotaxic exploration in frontal epilepsy. Adv Neurol 1992;57:651-88

32. Harvey AS, Hopkins IJ, Bowe JM, et al. Frontal lobe epilepsy: clinical seizure characteristics and localization with ictal 99mTc-HMPAO SPECT. Neurology 1993;43:1966-80

33. Lawson JA, Cook MJ, Vogrin S, et al. Clinical, EEG, and quantitative MRI differences in pediatric frontal and temporal lobe epilepsy. Neurology 2002;58:723-29

34. Braakman HM, Vaessen MJ, Jansen JF, et al. Pediatric frontal lobe epilepsy: white matter abnormalities and cognitive impairment. Acta Neurol Scand 2014;129:252-62 